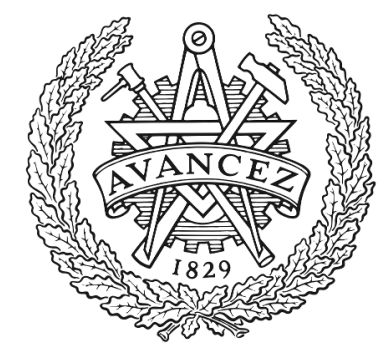

\title{
CHALMERS
}

UNIVERSITY OF TECHNOLOGY

\section{Robust inversion based fault estimation for discrete-time LPV systems}

Downloaded from: https://research.chalmers.se, 2023-04-26 06:34 UTC

Citation for the original published paper (version of record):

Kulcsár, B., Verhaegen, M. (2012). Robust inversion based fault estimation for discrete-time LPV systems. IEEE Transactions on Automatic Control, 57(6): 1581-1586.

http://dx.doi.org/10.1109/TAC.2011.2176160

N.B. When citing this work, cite the original published paper.

(C2012 IEEE. Personal use of this material is permitted.

However, permission to reprint/republish this material for advertising or promotional purposes 


\section{Robust Inversion Based Fault Estimation for Discrete-Time LPV Systems}

Balázs Kulcsár, Member, IEEE, and Michel Verhaegen

\begin{abstract}
The article presents a state-space based Fault Diagnosis (FD) method for discrete-time, affine Linear Parameter Varying (LPV) systems. The goal of the technical note is to develop a robust and dynamic inversion based technique for systems with parameter varying representations when an additive, exogenous disturbance signal perturbs the system. After applying geometric concepts for explicit fault inversion, a robust strategy is proposed to attenuate the effect of the unknown disturbance input signal on the fault estimation error. The proposed robust observer is derived as a solution of off-line Linear Matrix Inequality (LMI) conditions. The technical note demonstrates the viability of the novel methodology through a numerical example.
\end{abstract}

Index Terms-Discrete-time linear parameter varying (LPV) systems, dynamic inversion, fault detection and isolation, fault diagnosis (FD), robust observers.

\section{INTRODUCTION}

Fault Diagnosis (FD) is of capital importance in the design of complex and safety related systems. Since in FD recognition the occurring malfunction always conveys substantial information, the idea of reconstructing faults attracted significant research attention, e.g., [1]. Hence, instead of designing a controller robust enough to cover all possible effects of failures, FD techniques suggest the direct use of the detected fault signals, e.g. [2]. Even though, alternative nominal techniques have been developed, in most cases disturbance or modeling error do influence the quality of FD. Hence, various robust methods have been deployed, such as disturbance decoupling or other robust approaches, e.g. [3], [4]. FD for nonlinear systems has recently become an active research field. [5] gives an overview on nonlinear FD techniques considering both existing algebraic (e.g. [6]) and geometric approaches [7]. Besides, robust nonlinear FD has been identified as a relevant future research direction [5].

A rich set of linear methodologies has been developed for control, estimation and diagnosis of Linear Parameter Varying (LPV) model representations, such as [8]. LPV models with appropriate parametrization can be alternative to nonlinear system description. Methods for FD in LPV systems became attractive since they can significantly reduce computational complexity respectively result in explicit diagnosis solutions. In LPV FD literature, two main concepts have been proposed. First, indirect methods provide a residual signal (fault indicator). A nominal technique for continuous-time and affine LPV systems is described in [9]. Robust observer based indirect solutions are presented in [10]-[12]. Second, conceptually different (direct) approaches have been introduced in LPV fault estimation e.g. by means of nominal system inversion [13].

This technical note contributes to the field of fault estimation for discrete-time, affine LPV systems. Motivated by and connected to the प 口 प

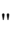

This work was supported by the Trans-

port Area of Advance at Chalmers University of Technology. Recommended by Associate Editor E. Weyer.

B. Kulcsár is with the Department of Signals and Systems, Chalmers University of Technology, Göteborg SE-412 96, Sweden (e-mail: kulcsar@chalmers. se).

M. Verhaegen is with the Delft Center for Systems and Control, Delft University of Technology, Delft 2628 CD, The Netherlands (e-mail: m.verhaegen@moesp.org). concise techniques in [9], [13], [14], this work presents a discrete-time and dynamic inversion based FD solution. The present methodology robustly reconstructs faults under the effect of unknown exogenous but norm bounded disturbance signals. This technical note, for a wide class of discrete-time LPV systems, allows one to consider a certain type of parameter variation at the plant output, as well as at the fault and disturbance input directions. Quadratically stabilizing and disturbance attenuating fault estimation is achieved by assigning a dynamic FD observer structure to the solution, namely a Luenberger type of observer. Even though, the original discrete-time LPV dynamics is affine in the scheduling parameter, the robust inversion procedure results in generic LPV model structures. Given the observer structure and the inverse system dynamics, the technical note shows how to attenuate disturbance signals on the performance output of the fault estimation problem, i.e. on the fault estimation error.

The technical note is divided into three main parts. After the introductory part, Section II formulates the robust and dynamic inversion problem to be solved. The main result of the technical note is discussed in Section III. The elaborated methodology is demonstrated by a numerical example in Section IV. Remarks and further concerns conclude the technical note. Technical derivations are tabulated to the Appendix.

\section{PROBLEM FORMULATION AND NOTATIONS}

Consider the following system by

$$
\begin{aligned}
x(k+1)= & \sum_{j=1}^{n p} p_{k}^{(j)}\left(A^{(j)} x(k)+B_{2}^{(j)} u(k)\right. \\
& \left.+E^{(j)} f(k)+B_{1}^{(j)} d(k)\right) \\
y(k)= & \sum_{j=1}^{n p} p_{k}^{(j)}\left(C_{2}^{(j)} x(k)+D_{22}^{(j)} u(k)+D_{21}^{(j)} d(k)\right)
\end{aligned}
$$

where $f, u, x, y, d, p \in \mathbb{R}^{n f, m, n, \ell, n^{d, n} p}$ are the fault, control input, state, output, disturbance, and scheduling parameter signals with appropriate dimensional, known, and real valued state-space matrices $A^{(j)}, E^{(j)}, B_{1}^{(j)}, B_{2}^{(j)}, C_{2}^{(j)}, D_{21}^{(j)}, D_{22}^{(j)}, j=1 \ldots n_{p}$ with $p^{(1)}=1$ and $\ell \geq n_{f}$. The scheduling parameter $p$ belongs to a compact polytope $\mathcal{P} \subseteq \mathbb{R}^{n^{p}}$ with vertices $\overline{\mathcal{P}}$ defined by $p \in[p \bar{p}]$. In case of the sensor fault, we suggest to use pseudo actuation [15] in order to yield the above form. Up to the time sample $k$, the fault-free $p_{k}{ }^{1}$, $u(k)$, and $y(k)$ are measurable but the fault input $f(k)$ and the 2-norm bounded disturbance signal $d(k)$ are unknown.

For the sake of simplicity, we assume in the remaining part of Section II that $u(k)=d(k)=0$. The goal of the problem statement section is to show how to express the fault signal $f(k)$ purely by measurable quantities and model parameter information.

Assumption 1: We assume, the kernel space of the affine parameter dependent output map, is parameter independent (constant) and will be denoted by $\mathcal{C}=\operatorname{ker}\left\{\sum_{j=1}^{n} p_{k}^{(j)} C_{2}^{(j)}\right\}, \forall p_{k} \in \mathcal{P}, \forall k \in \mathbb{Z}$. In other words, the scheduling trajectories are such that they never cause any changes in the kernel space directions of the measurement map, though, the output (2) is parameter dependent.

Assumption 2: $\sum_{j=1}^{n^{p}} p_{k}^{(j)} E^{(j)}$ is full column rank $\forall p_{k} \in \mathcal{P}$. Alternatively, the kernel space of the fault insertion map is the origin for all possible parameter trajectories.

In the sequel, an algorithm is provided to show how to over-parametrize the parameter varying fault direction.

${ }^{1}$ Sensors used for the measurement of the scheduling parameters have to be free of direct fault. In case of endogenous parameters, we do not preclude implicit fault conditions until $p^{k}$ reflects the true system behavior. 
Algorithm 1:

Step 1 Consider $\sum_{j=1}^{n_{p}} p_{k}^{(j)} E^{(j)} f(k)=E \tilde{f}(k), \tilde{f}(k) \in \mathbb{R}^{n_{\tilde{f}}}$ with $\mathcal{E}=\operatorname{im}\left\{\bigoplus_{j=1}^{n p} E^{(j)}\right\}$ where $\bigoplus$ and $\operatorname{im}\{\cdot\}$ denote the subspace dilatation and the computation of the range space, respectively. $E$ is an orthonormal basis representation of $\mathcal{E}$.

Step 2 In virtue of Assumption 2, $\tilde{f}(k)$ can be unambiguously projected back to $f(k)$ by $P(k)=\left(\sum_{j=1}^{n_{p}} p_{k}^{(j)} E^{(j)}\right)^{\dagger} E$, where $(\cdot)^{\dagger}$ stands for the pseudo inverse.

Assumption 3: System (1), (2) is reversible [16], [17], quadratically detectable [18] and (strong) invertible, i.e. $\mathcal{R}_{E}^{*}=\{0\}$ [19], with parameter invariant relative degree vector. $\mathcal{R}_{E}^{*}$ is the largest fault controllability subspace in the kernel space of the output map $\mathcal{C}$.

Lemma 1: Given the system in (1)-(2) with $u(k)=d(k)=0$. If Assumptions 1-3. are fulfilled, based on Algorithm 1, the inverse system dynamics can be written as

$$
\begin{aligned}
x_{2}(k+1) & =\mathcal{A}(k) x_{2}(k)+\mathcal{B}(k+\kappa) \mathbf{y}_{[k, k+\kappa)} \\
f(k) & =\mathcal{C}(k) x_{2}(k)+\mathcal{D}(k+\kappa) \mathbf{y}_{[k, k+\kappa)}
\end{aligned}
$$

where the coefficient matrices $\mathcal{A}(k), \mathcal{B}(k+\kappa), \mathcal{C}(k), \mathcal{D}(k+\kappa)$ (see (16), (17)) are generic parameter varying coefficient matrices. Furthermore, $x_{2}(k)$ characterizes the dynamics within the largest fault $\left(\sum_{j=1}^{n_{p}} p_{k}^{(j)} A^{(j)}, \sum_{j=1}^{n_{p}} p_{k}^{(j)} E^{(j)}\right)$ invariant subspace contained in $\mathcal{C}$, and $\mathbf{y}_{[k, k+\kappa)}$ is the measured output vector along the future time horizon up to $\kappa$ denoting the maximal element of the relative degree vector.

Proof: $\mathcal{R}_{E}^{*}$ can be constructed by [14], based on the fault Controllability Subspace Algorithm starting from the largest $\left(\sum_{j=1}^{n_{p}} p_{k}^{(j)} A^{(j)}, \sum_{j=1}^{n_{p}} p_{k}^{(j)} E^{(j)}\right)$ invariant subspace denoted by $\mathcal{V}^{*} \subseteq \mathcal{C}$. The invertibility condition in Assumption 3 can be reformulated as a left invertibility condition by $\mathcal{V}^{*} \cap \mathcal{E}=\{0\}$. The left invertibility condition then gives rise to a nonsingular, parameter independent coordinate transformation [13], $T: \mathbb{R}^{n} \mapsto \mathbb{R}^{n}$ splits the original LPV representation into two parts. $T=\left[V^{*, \perp} V^{*}\right]$, where $V^{*, \perp}$ and $V^{*}$ are bases for the orthogonal complement of $\mathcal{V}^{*}$ denoted by $\mathcal{V}^{*, \perp}$, and for $\mathcal{V}^{*}$, respectively. First, we apply Step 1 of Algorithm 1 , then $T$ to the original system in (1)-(2).

Then, the following form can be obtained:

$$
\begin{aligned}
x_{1}(k+1) & =\sum_{j=1}^{n_{p}} p_{k}^{(j)}\left(A_{11}^{(j)} x_{1}(k)+A_{12}^{(j)} x_{2}(k)\right)+E_{1} \tilde{f}(k) \\
x_{2}(k+1) & =\sum_{j=1}^{n_{p}} p_{k}^{(j)}\left(A_{21}^{(j)} x_{1}(k)+A_{22}^{(j)} x_{2}(k)\right) \\
y(k) & =\sum_{j=1}^{n_{p}} p_{k}^{(j)} \tilde{C}_{2}^{(j)} x_{1}(k)
\end{aligned}
$$

where the LPV system is decomposed in two interconnected dynamics, $x_{1}$ characterizes the dynamics in $\mathcal{V}^{*, \perp}$ with $\operatorname{dim}\left(\mathcal{V}^{*, \perp}\right)=n_{\kappa}$ and $x_{2}$ refers to the dynamics in $\mathcal{V}^{*}$ with $\operatorname{dim}\left(\mathcal{V}^{*}\right)=n-n_{\kappa}$.

With an appropriately chosen feedback term $F_{2}^{(j)}, A_{12}^{(j)}$ can be naturally canceled out by preserving invariance [13] if

$$
\tilde{f}(k)=\left[\begin{array}{ll}
0 & \sum_{j=1}^{n_{p}} p_{k}^{(j)} F_{2}^{(j)}
\end{array}\right]\left[\begin{array}{l}
x_{1}(k) \\
x_{2}(k)
\end{array}\right]+f_{r}(k) .
$$

In this case, $E_{1} F_{2}^{(j)}=-A_{12}^{(j)}$ is an obvious choice and $f_{r}(k)$ represents a synthetic fault signal. Thus, substituting (8) into (5) reads as

$$
x_{1}(k+1)=\sum_{j=1}^{n_{p}} p_{k}^{(j)} A_{11}^{(j)} x_{1}(k)+E_{1} f_{r}(k)
$$

$$
\begin{aligned}
x_{2}(k+1) & =\sum_{j=1}^{n_{p}} p_{k}^{(j)}\left(A_{21}^{(j)} x_{1}(k)+A_{22}^{(j)} x_{2}(k)\right) \\
y(k) & =\sum_{j=1}^{n_{p}} p_{k}^{(j)} \tilde{C}_{2}^{(j)} x_{1}(k) .
\end{aligned}
$$

Unlike the subsystem in (10), the dynamics in (9) can be completely characterized by the output measurement (11) and the scheduling parameter [14]. $f_{r}(k)$ can therefore be reconstructed simply based on measurable information. Therefore, denote the rows of the output map $\tilde{C}_{2}^{(j)} \forall j=1 \ldots n_{p}$ by $c_{i}^{(j)}, i=1 \ldots \ell$. Define $\kappa_{i}$, the relative degree of the $i^{t h}$ output channel based on Algorithm 2., $\tilde{\kappa}=\left[\begin{array}{lllll}\kappa_{1} & \ldots & \kappa_{i} & \ldots & \kappa_{\ell}\end{array}\right]$ (see Appendix for details) $)^{2}$ Thus, $y_{i}(k)=\sum_{j=1}^{n_{p}} p_{k}^{(j)} c_{i}^{(j)} x_{1}(k), \ldots, y_{i}\left(k+\kappa_{i}-\right.$ 1) $=\sum_{j=1}^{n p} p_{k+\kappa_{i}-1}^{(j)} c_{i}^{(j)} \sum_{j=1}^{n p} p_{k+\kappa_{i}-2}^{(j)} A_{11}^{(j)} \cdots \sum_{j=1}^{n_{p}} p_{k}^{(j)} A_{11}^{(j)} x_{1}(k)$, where we collect output information as long as we do not exceed sample $\kappa_{i}-1$.

Condensing the measurements of the output channel $i$ into a vector $\mathbf{y}$ results in

$$
\mathbf{y}_{\left[k, k+\kappa_{i}-1\right)}=\mathcal{O}_{\left[k, k+\kappa_{i}-1\right)} x_{1}(k) .
$$

Repeating the above procedure for all output channels, we can write

$$
\mathbf{y}_{[k, k+\kappa-1)}=\left[\begin{array}{c}
\mathbf{y}_{\left[k, k+\kappa_{1}-1\right)} \\
\vdots \\
\mathbf{y}_{\left[k, k+\kappa_{\ell}-1\right)}
\end{array}\right]=\mathcal{O}_{[k, k+\kappa-1)} x_{1}(k) .
$$

Note that in output segment $\mathbf{y}_{[k, k+\kappa-1)}$ no complete information will appear on $f_{r}(k)$, in virtue of the definition of $\tilde{\kappa}$ and $\kappa$. Now, let us define the vector

$$
\tilde{\mathcal{E}}_{\left[k, k+\kappa_{i}\right)}=\sum_{j=1}^{n_{p}} p_{k+\kappa_{i}}^{(j)} c_{i}^{(j)} \cdot \sum_{j=1}^{n_{p}} p_{k+\kappa_{i}-1}^{(j)} A_{11}^{(j)} \cdots \sum_{j=1}^{n_{p}} p_{k+1}^{(j)} A_{11}^{(j)} E_{1}
$$

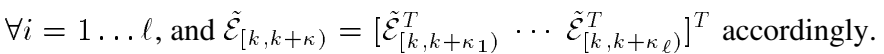
At time sample $k+\kappa$, the effect of the fault term $f_{r}(k)$ appears in the output equation under the form of

$$
\mathbf{y}_{[k, k+\kappa)}=\mathcal{O}_{[k, k+\kappa)} x_{1}(k)+\tilde{\mathcal{E}}_{[k, k+\kappa)} f_{r}(k) .
$$

Consequently, by substituting the observations from (13) into (14) if $\sum_{l=1}^{\ell} \kappa_{l} \geq n-n_{\kappa}$ and expressing $f_{r}(k)$, we can write

$$
f_{r}(k)=\tilde{\mathcal{E}}_{[k, k+\kappa)}^{\dagger}\left(\mathbf{y}_{[k, k+\kappa)}-\mathcal{O}_{[k, k+\kappa)} \mathcal{O}_{[k, k+\kappa-1)}^{\dagger} \mathbf{y}_{[k, k+\kappa-1)}\right) .
$$

Now, the dynamics of the inverse system w.r.t the fault input can be read as a non-affine LPV system defined between the new system inputs $\mathbf{y}_{[k, k+\kappa)}$ and the inverse system's output $f(k)$. Recall the system in equations (9)-(11) and replace the state vector $x_{1}(k)$ by the measurements based on (13).

The dynamics of the inverse system is charaterized by (10) and can be rewritten into the form of (16), respectively. Furthermore, the combination of (15) and the feedback term in eq. (8) together with Step 2 from Algorithm 1 results in (17). Accordingly

$$
x_{2}(k+1)=\underbrace{\sum_{j=1}^{n_{p}} p_{k}^{(j)} A_{22}^{(j)}}_{\mathcal{A}(k)} x_{2}(k)+\underbrace{\sum_{j=1}^{n_{p}} p_{k}^{(j)} A_{21}^{(j)} \mathcal{O} c_{[k+\kappa-1)}^{\dagger} \mathcal{S}_{y}}_{\mathcal{B}(k+\kappa)} \mathbf{y}_{[k, k+\kappa)}
$$

${ }^{2} \kappa_{i} \forall i=1 \ldots \ell$ is assumed to be constant for all possible scheduling trajectory in any time interval. 


$$
\begin{aligned}
& f(k)=\underbrace{-P(k) \sum_{j=1}^{n_{p}} p_{k}^{(j)}\left(E_{1}\right)^{\dagger} A_{12}^{(j)}}_{\mathcal{C}(k)} x_{2}(k) \\
& +\underbrace{P(k) \tilde{\mathcal{E}}_{[k, k+\kappa-1)}^{\dagger}\left(\mathcal{I}_{\kappa_{s}}-\mathcal{O}_{[k, k+\kappa)} \mathcal{O}_{[k, k+\kappa-1)}^{\dagger} \mathcal{S}_{y}\right)}_{\mathcal{D}(k+\kappa)} \mathbf{y}_{[k, k+\kappa)}
\end{aligned}
$$

with the selector $\mathcal{S}_{y}$ defined as $\mathbf{y}_{[k, k+\kappa-1)}=\mathcal{S}_{y} \mathbf{y}_{[k, k+\kappa)} . \mathcal{I}_{\kappa_{s}}$ stands for the identity matrix with dimension $\kappa_{s}=\sum_{l=1}^{\ell} \kappa_{l} 3$ This completes the proof.

The above-mentioned nominal procedure for discrete-time LPV fault inversion might be sensitive to exogenous disturbance signals. If complete disturbance decoupling is not possible, this technical note shows an efficient way for inversion based and disturbance rejecting observer design via a Luenberger observer. We shall refer to matrix inequalities by the symbol $\mathcal{A} \succ 0$, denoting that matrix $\mathcal{A}$ is positive definite. The disturbance signal $d(k)$ with bounded 2-norm is denoted by $\|d\|_{2}=\left(\sum_{k=0}^{\infty} d(k)^{T} d(k)\right)^{1 / 2}$ and $\mathbf{d}_{\left[k, k+k_{1}\right)}=\left[d(k)^{T} \ldots d\left(k+k_{1}\right)^{T}\right]^{T}$. The superscript $(\cdot)^{T}$ denotes the transpose of the real matrix and $\star$ represents the symmetric term of the matrix inequality.

\section{RoBUSt FAULT INVERSION}

The following Lemma extends the results of Lemma 1., first, by considering non-zero input and disturbance signals. Second, it explains the way of obtaining a system description for robust and model-based observer design.

Lemma 2: Given the system in equations (1)-(2). If Assumptions 1-3. are fulfilled, based on Algorithm 1, the inverse system dynamics can written as

$$
\begin{aligned}
& {\left[\begin{array}{c}
x(k-\kappa+1) \\
f(k-\kappa) \\
y(k-\kappa)
\end{array}\right]} \\
& =\left[\begin{array}{c|cc}
\mathcal{A}(k) & \mathcal{B}_{1}(k) & \mathcal{B}_{2}(k) \\
\hline \mathcal{C}_{1}(k) & \mathcal{D}_{11}(k) & \mathcal{D}_{12}(k) \\
\mathcal{C}_{2}(k-\kappa) & \mathcal{D}_{21}(k-\kappa) & \mathcal{D}_{22}(k-\kappa)
\end{array}\right]\left[\begin{array}{c}
x(k-\kappa) \\
\mathbf{d}_{[k-\kappa, k)} \\
\tilde{\mathbf{u}}_{[k-\kappa, k)}
\end{array}\right]
\end{aligned}
$$

where the coefficient matrices $\mathcal{A}(k), \mathcal{B}(k), \mathcal{C}_{1}(k), \mathcal{D}_{11}(k)$, $\mathcal{D}_{12}(k), \mathcal{C}_{2}(k-\kappa), \mathcal{D}_{21}(k-\kappa), \mathcal{D}_{22}(k-\kappa)$, [see (28)-(31)] are generic parameter varying coefficients. Furthermore, $\mathbf{d}_{[k-\kappa, k)}$ and $\tilde{\mathbf{u}}_{[k-\kappa, k)}=\left[y(k)^{T} \mathbf{u}_{[k-\kappa, k)}^{T}\right]^{T}$ are the vectorized values of

${ }^{3}$ In case of discrete-time LPV inversion, one both needs the forward time shifted output and scheduling parameter values with at least $\kappa=\max \{\tilde{\kappa}\}$ samples. Consequently, if we have causal measurements the system can only be inverted with a time delay $\kappa$. disturbance and of the augmented input signals for the time interval $k-\kappa$ to $k$, respectively.

Proof: Recall the system representation in (1)-(2) with Step 1 of Algorithm 1 and apply the coordinate transformation $T$ with the feedback design in (8), then

$$
\begin{aligned}
x_{1}(k+1)= & \sum_{j=1}^{n_{p}} p_{k}^{(j)}\left(A_{11}^{(j)} x_{1}(k)+B_{12}^{(j)} u(k)+B_{11}^{(j)} d(k)\right) \\
& +E_{1} f_{r}(k) \\
x_{2}(k+1)= & \sum_{j=1}^{n_{p}} p_{k}^{(j)}\left(A_{21}^{(j)} x_{1}(k)+A_{22}^{(j)} x_{2}(k)+B_{22}^{(j)} u(k)\right. \\
& \left.+B_{21}^{(j)} d(k)\right) \\
y(k)= & \sum_{j=1}^{n_{p}} p_{k}^{(j)}\left(\tilde{C}_{2}^{(j)} x_{1}(k)+D_{22}^{(j)} u(k)\right. \\
& \left.+D_{21}^{(j)} d(k)\right) .
\end{aligned}
$$

Denote the rows of $\tilde{C}_{2}^{(j)}$ by $c_{i}^{(j)}$ and of $D_{22}^{(j)}$ and $D_{21}^{(j)}$ by $d_{21, i}^{(j)}$ and $d_{22, i}^{(j)}$ where $i=1 \ldots \ell, j=1 \ldots n_{p}$. Define

$$
\begin{aligned}
\mathcal{O}_{[k, k+\kappa)}= & {\left[\begin{array}{c}
\sum_{j=1}^{n_{p}} p_{k+\kappa_{1}}^{(j)} c_{1}^{(j)} \cdots \sum_{j=1}^{n_{p}} p_{k}^{(j)} A_{11}^{(j)} \\
\vdots \\
\sum_{j=1}^{n_{p}} p_{k+\kappa_{\ell}}^{(j)} c_{\ell}^{(j)} \cdots \sum_{j=1}^{n_{p}} p_{k}^{(j)} A_{11}^{(j)}
\end{array}\right] } \\
\tilde{\mathcal{E}}_{[k, k+\kappa)}= & {\left[\begin{array}{c}
\sum_{j=1}^{n_{p}} p_{k+\kappa_{1}}^{(j)} c_{1}^{(j)} \cdots \sum_{j=1}^{n_{p}} p_{k+1}^{(j)} A_{11}^{(j)} E_{1} \\
\vdots \\
\sum_{j=1}^{n_{p}} p_{k+\kappa_{\ell}}^{(j)} c_{\ell}^{(j)} \cdots \sum_{j=1}^{n_{p}} p_{k+1}^{(j)} A_{11}^{(j)} E_{1}
\end{array}\right] . }
\end{aligned}
$$

In (24) and (25), shown at the bottom of the page, $O_{\kappa-\kappa_{i}}$ is a zero block and $\kappa$ is the largest relative degree number $\forall i=1 \ldots \ell$. Therefore ${ }^{4}$

$$
\begin{aligned}
y(k+\kappa)= & \mathcal{O}_{[k, k+\kappa)} x_{1}(k)+\mathcal{T}_{u,[k, k+\kappa)} \mathbf{u}_{[k, k+\kappa)} \\
& +\mathcal{T}_{d,[k, k+\kappa)} \mathbf{d}_{[k, k+\kappa)}+\tilde{\mathcal{E}}_{[k, k+\kappa)} f_{r}(k) \\
f_{r}(k)= & \tilde{\mathcal{E}}_{[k, k+\kappa)}^{-\ell}\left(y(k+\kappa)-\mathcal{O}_{[k, k+\kappa)} x_{1}(k)\right. \\
& +\mathcal{T}_{u,[k, k+\kappa)} \mathbf{u}_{[k, k+\kappa)} \\
& \left.-\mathcal{T}_{d,[k, k+\kappa)} \mathbf{d}_{[k, k+\kappa)}\right) .
\end{aligned}
$$

Together with the dynamic evolution of $x_{2}$ in (20), (27) defines the inverse system w.r.t. the synthetic fault input $f_{r}(k)$. Instead of directly using the inverse system for robust detection filter design, we revert to

${ }^{4}$ In (24)-(25) the amount of information (i.e. the number of $u$ and $d$ samples) influencing the output at time sample $y(k+\kappa)$ is changing due to fact that $\kappa_{i}$ is different $\forall i=1 \ldots \ell$. In fact, the Toeplitz structures in (24)-(25), shown at the bottom of the page, might have zero padded columns depending on $\kappa_{i}$ as well as on the relative degree vectors associated to the input and disturbance terms.

$$
\begin{aligned}
\mathcal{T}_{d,[k, k+\kappa)}= & {\left[\begin{array}{ccccc}
\sum_{j=1}^{n_{p}} p_{k+\kappa_{1}}^{(j)} c_{1}^{(j)} \cdot \sum_{j=1}^{n_{p}} p_{k+\kappa_{1}-1}^{(j)} A_{11}^{(j)} \cdots \sum_{j=1}^{n_{p}} p_{k}^{(j)} B_{11}^{(j)} & \cdots & \sum_{j=1}^{n_{p}} p_{k+\kappa_{1}}^{(j)} d_{21, \ell}^{(j)} & O_{\kappa-\kappa_{1}} \\
\vdots & & & \\
\sum_{j=1}^{n_{p}} p_{k+\kappa_{\ell}}^{(j)} c_{\ell}^{(j)} \cdot \sum_{j=1}^{n_{p}} p_{k+\kappa_{\ell}-1}^{(j)} A_{11}^{(j)} \cdots \sum_{j=1}^{n_{p}} p_{k}^{(j)} B_{11}^{(j)} & \cdots & \sum_{j=1}^{n_{p}} p_{k+\kappa_{\ell}}^{(j)} d_{21, \ell}^{(j)} & O_{\kappa-\kappa_{\ell}}
\end{array}\right] } \\
\mathcal{T}_{u,[k, k+\kappa)}= & {\left[\begin{array}{ccccc}
\sum_{j=1}^{n_{p}} p_{k+\kappa_{1}}^{(j)} c_{1}^{(j)} \cdot \sum_{j=1}^{n_{p}} p_{k+\kappa_{1}-1}^{(j)} A_{11}^{(j)} \cdots \sum_{j=1}^{n_{p}} p_{k}^{(j)} B_{12}^{(j)} & \cdots & \sum_{j=1}^{n} p_{k+\kappa_{1}}^{(j)} d_{22, \ell}^{(j)} & O_{\kappa-\kappa_{1}} \\
\vdots & & & \\
\sum_{j=1}^{n_{p}} p_{k+\kappa_{\ell}}^{(j)} c_{\ell}^{(j)} \cdot \sum_{j=1}^{n_{p}} p_{k+\kappa_{\ell}-1}^{(j)} A_{11}^{(j)} \cdots \sum_{j=1}^{n_{p}} p_{k}^{(j)} B_{12}^{(j)} & \cdots & \sum_{j=1}^{n_{p}} p_{k+\kappa_{\ell}}^{(j)} d_{22, \ell}^{(j)} & O_{\kappa-\kappa_{\ell}}
\end{array}\right] }
\end{aligned}
$$


the original system description, (19)-(21). By feeding back the information $f_{r}(k)$ (and using the Step 2 in Algorithm 1) as well as considering $\tilde{\mathbf{u}}_{[k, k+\kappa)}=\left[y(k+\kappa)^{T} \mathbf{u}_{[k, k+\kappa)}^{T}\right]^{T}$, we can write

$$
\begin{aligned}
& x_{1}(k+1)=\left(\sum_{j=1}^{n_{p}} p_{k}^{(j)} A_{11}^{(j)}-E_{1} \tilde{\mathcal{E}}_{[k, k+\kappa)}^{-\ell} \mathcal{O}_{[k, k+\kappa)}\right) x_{1}(k) \\
& +\left[\sum_{j=1}^{n_{p}} p_{k}^{(j)} B_{11}^{(j)}-E_{1} \tilde{\mathcal{E}}_{[k, k+\kappa)}^{-\ell} \mathcal{T}_{d,[k, k+\kappa)}\right] \\
& \times\left[\begin{array}{c}
d(k) \\
\mathbf{d}_{[k, k+\kappa)}
\end{array}\right]+E_{1} \tilde{\mathcal{E}}_{[k, k+\kappa)}^{-\ell} y(k+\kappa) \\
& +\sum_{j=1}^{n_{p}} p_{k}^{(j)} B_{21}^{(j)} u(k) \\
& -E_{1} \tilde{\mathcal{E}}_{[k, k+\kappa)}^{-\ell} \mathcal{T}_{u,[k, k+\kappa)} \mathbf{u}_{[k, k+\kappa)} \\
& =\mathcal{A}_{11}(k+\kappa) x_{1}(k) \\
& +\mathcal{B}_{11}(k+\kappa) \mathbf{d}_{[k, k+\kappa)} \\
& +\mathcal{B}_{12}(k+\kappa) \tilde{\mathbf{u}}_{[k, k+\kappa)} \\
& x_{2}(k+1)=\sum_{j=1}^{n_{p}} p_{k}^{(j)}\left(A_{21}^{(j)} x_{1}(k)+A_{22}^{(j)} x_{2}(k)\right. \\
& \left.+B_{21}^{(j)} d(k)+B_{22}^{(j)} u(k)\right) \\
& =\mathcal{A}_{21}(k) x_{1}(k)+\mathcal{A}_{22}(k) x_{2}(k) \\
& +\mathcal{B}_{21}(k) \mathbf{d}_{[k, k+\kappa)}+\mathcal{B}_{22}(k) \tilde{\mathbf{u}}_{[k, k+\kappa)} \\
& y(k)=\sum_{j=1}^{n_{p}} p_{k}^{(j)}\left(\tilde{C}_{2}^{(j)} x_{1}(k)+D_{22}^{(j)} u(k)+D_{21}^{(j)} d(k)\right) \\
& =\tilde{\mathcal{C}}_{2}(k) x_{1}(k)+\mathcal{D}_{22}(k) \tilde{\mathbf{u}}_{[k, k+\kappa)} \\
& +\mathcal{D}_{21}(k) \mathbf{d}_{[k, k+\kappa)}
\end{aligned}
$$

where $\tilde{\mathbf{u}}_{[k, k+\kappa)} \in \mathbb{R}^{\tilde{m}}, \tilde{m}=\sum_{i=1}^{\ell}\left(\kappa_{i} \cdot m\right)+\ell$ and $\mathbf{d}_{[k, k+\kappa)} \in \mathbb{R}^{\tilde{n} d}$, $\tilde{n}_{d}=\sum_{i=1}^{\ell}\left(\kappa_{i} \cdot n_{d}\right), x_{1} \in \mathbb{R}^{n^{-n} n_{\kappa}}$ and $x_{2} \in \mathbb{R}^{n_{\kappa}}$.

In addition, we define $f(k)$ as a performance output of the inverse system by

$$
\begin{aligned}
f(k)= & P(k)\left(f_{r}(k)+\sum_{j=1}^{n_{p}} p_{k}^{(j)} F_{2}^{(j)} x_{2}(k)\right) \\
= & -P(k) \tilde{\mathcal{E}}_{[k, k+\kappa)}^{-\ell} \mathcal{O}_{[k, k+\kappa)} x_{1}(k) \\
& +P(k) \sum_{j=1}^{n_{p}} p_{k}^{(j)} F_{2}^{(j)} x_{2}(k) \\
& +\left[P(k) \tilde{\mathcal{E}}_{[k, k+\kappa)}^{-\ell}-P(k) \tilde{\mathcal{E}}_{[k, k+\kappa)}^{-\ell} \mathcal{T}_{u,[k, k+\kappa)}\right] \\
& \times[y(k+\kappa)] \\
& -P(k) \tilde{\mathcal{E}}_{[k, k+\kappa)}^{-\ell} \mathcal{T}_{d,[k, k+\kappa)} \mathbf{d}_{[k, k+\kappa)} \\
= & \mathcal{C}_{11}(k+\kappa) x_{1}(k) \\
& +\mathcal{C}_{12}(k) x_{2}(k)+\mathcal{D}_{11}(k+\kappa) \mathbf{d}_{[k, k+\kappa)} \\
& +\mathcal{D}_{12}(k+\kappa) \tilde{\mathbf{u}}_{[k, k+\kappa)} .
\end{aligned}
$$

In case of causal measurements, we can shift the future sample back to the current time instant and write

$$
\mathcal{X}=\left[\begin{array}{c}
x_{1}(k-\kappa+1) \\
x_{2}(k-\kappa+1) \\
f(k-\kappa) \\
y(k-\kappa)
\end{array}\right], \quad \mathcal{Y}=\left[\begin{array}{c}
x_{1}(k-\kappa) \\
x_{2}(k-\kappa) \\
\mathbf{d}_{[k-\kappa, k)} \\
\tilde{\mathbf{u}}_{[k-\kappa, k)}
\end{array}\right]
$$

$$
\mathcal{X}=\left[\begin{array}{cc|cc}
\mathcal{A}_{11}(k) & O_{n_{\kappa}} & \mathcal{B}_{11}(k) & \mathcal{B}_{12}(k) \\
\mathcal{A}_{21}(k-\kappa) & \mathcal{A}_{22}(k-\kappa) & \mathcal{B}_{21}(k-\kappa) & \mathcal{B}_{22}(k-\kappa) \\
\hline \mathcal{C}_{11}(k) & \mathcal{C} c_{12}(k-\kappa) & \mathcal{D}_{11}(k) & \mathcal{D}_{12}(k) \\
\tilde{\mathcal{C}}_{2}(k-\kappa) & O_{n_{\kappa}} & \mathcal{D}_{21}(k-\kappa) & \mathcal{D}_{22}(k-\kappa)
\end{array}\right] \mathcal{Y}
$$

which form directly implies equations (19)-(21). This completes the proof.

In system representation (19)-(21), reconstruction of $f(k)$ will be effected by $\mathbf{d} \neq 0$. Hence, in the sequel, we suggest to reject the disturbance on the fault estimation error up to a certain performance level $\gamma>0$. We propose to formulate a quadratic objective function and minimize the effect of $\mathbf{d} \in \mathbb{R}^{n} \mathbf{d}$ on $\Delta f=f-\hat{f}$

$$
\|(f(k-\kappa)-\hat{f}(k-\kappa))\|_{2}-\gamma^{2}\left\|\mathbf{d}_{[k-\kappa, k)}\right\|_{2}<0
$$

$\forall p_{k} \in \mathcal{P}$ with a scalar $\gamma>0$. This objective can be achieved by assigning a structure to the robust fault estimation problem.

In the present technical note, we apply a Luenberger observer by

$$
\begin{aligned}
\hat{x}(k-\kappa+1)= & \mathcal{A}(k) \hat{x}(k-\kappa)+\mathcal{B}_{2}(k) \tilde{\mathbf{u}}_{[k-\kappa, k)} \\
& +\mathcal{K}(k)[y(k-\kappa)-\hat{y}(k-\kappa)] \\
\hat{y}(k-\kappa)= & \mathcal{C}_{2}(k-\kappa) \hat{x}(k+\kappa)+\mathcal{D}_{22}(k-\kappa) \tilde{\mathbf{u}}_{[k-\kappa, k)} \\
\hat{f}(k-\kappa)= & \mathcal{C}_{1}(k) \hat{x}(k+\kappa)+\mathcal{D}_{12}(k-\kappa) \tilde{\mathbf{u}}_{[k-\kappa, k)}
\end{aligned}
$$

where we have a large freedom to select the structure behind the gain matrix $\mathcal{K}(k)$. With the help of affine gain structure, we can involve past scheduling parameters under the form of $\mathcal{K}(k)=\mathcal{K}^{(1)}+\sum_{j=0}^{\kappa} \sum_{i=2}^{n p} p_{k-j}^{(i)} \mathcal{K}_{j}^{(i)}$. Apart the fulfillment of the predefined objective in inequality (33), the closed-loop observer has to preserve asymptotic stability. Note that the above criteria fit to the generic problem formulation of $\mathcal{H}_{\infty}$ smoothing [20], or delayed $\mathcal{H}_{\infty}$ filter design [21]. As a result of Lemma 2, the inverse system naturally structures delays when replacing the fault input with measurement. Moreover, throughout $\mathcal{K}(k)$, we encounter past scheduling measurements in the robust observer synthesis.

Theorem 1: Given the system in (18), the quadratic objective function in (33) together with the observer structure in (34)-(36). With $\mathcal{K}(k)=\mathcal{K}^{(1)}+\sum_{j=0}^{\kappa} \sum_{i=2}^{n p} p_{k-j}^{(i)} \mathcal{K}_{j}^{(i)}$ the proposed observer results in a asymptotically unbiased estimate of $f$ when $\mathbf{d}=0$, furthermore, in case of $\mathbf{d} \neq 0$, it attenuates in induced $\mathcal{L}_{2}$ norm sense the disturbance on the fault estimation error $\Delta f$ up to a given $\gamma>0$ with $G \succ 0$ if the following matrix inequalities are feasible:

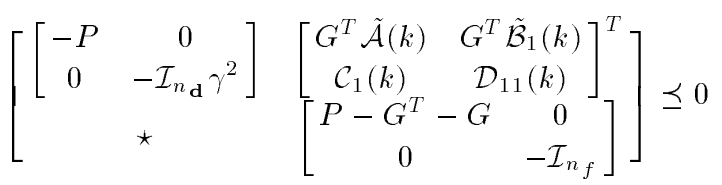

with $P=P^{T} \succ 0$ and $\tilde{\mathcal{A}}(k)=\mathcal{A}(k)-\mathcal{K}(k) \mathcal{C}_{2}(k-\kappa)$ and $\mathcal{B}_{1}(k)+$ $\mathcal{K}(k) \mathcal{D}_{21}(k-\kappa)$.

Proof: Consider the dynamics of the state reconstruction error by

$$
\begin{aligned}
e_{x}(k-\kappa+1)= & \underbrace{\left(\mathcal{A}(k)-\mathcal{K}(k) \mathcal{C}_{2}(k-\kappa)\right)}_{\tilde{\mathcal{A}}(k)} e_{x}(k-\kappa) \\
& +\underbrace{\left(\mathcal{B}_{1}(k)+\mathcal{K}(k) \mathcal{D}_{21}(k-\kappa)\right)}_{\tilde{\mathcal{B}}_{1}(k)} \mathbf{d}_{[k-\kappa, k)} \\
\Delta f(k-\kappa)= & f(k-\kappa)-\hat{f}(k-\kappa) \\
= & \mathcal{C}_{1}(k) e_{x}(k-\kappa)+\mathcal{D}_{11}(k) \mathbf{d}_{[k-\kappa, k)} .
\end{aligned}
$$


Define a Lyapunov function with constant $P^{T}=P \succ 0$ for the state reconstruction error by

$$
V(k-\kappa)=e_{x}(k-\kappa)^{T} P e_{x}(k-\kappa)>0, \quad e_{x}(k-\kappa) \neq 0 .
$$

Consequently, to achieve asymptotic stability, we intend to find $P$ and $\mathcal{K}(k)$ based on the dissipativity condition and the performance objective [defined in (33)]

$$
\begin{aligned}
& V(k-\kappa+1)-V(k-\kappa)+\Delta f(k-\kappa)^{T} \Delta f(k-\kappa) \\
& -\gamma^{2} \mathbf{d}_{[k-\kappa, k)}^{T} \mathbf{d}_{[k-\kappa, k)} \\
& =e_{x}(k-\kappa+1)^{T} P e_{x}(k-\kappa+1)^{T} \\
& \quad-e_{x}(k-\kappa)^{T} P e_{x}(k-\kappa)+\Delta f(k-\kappa)^{T} \Delta f(k-\kappa)- \\
& \quad-\gamma^{2} \mathbf{d}_{[k-\kappa, k)}^{T} \mathbf{d}_{[k-\kappa, k)} \leq 0 .
\end{aligned}
$$

By using the dynamics of the error equation and rearranging the terms, we can write

$$
\begin{aligned}
& \mathcal{L}_{1}=\tilde{\mathcal{A}}(k)^{T} P \tilde{\mathcal{A}}(k)-P+\mathcal{C}_{1}(k)^{T} \mathcal{C}_{1}(k) \\
& \mathcal{L}_{2}=\tilde{\mathcal{A}}(k)^{T} P \tilde{\mathcal{B}}_{1}(k)+\mathcal{C}_{1}(k)^{T} \mathcal{D}_{11}(k) \\
& \mathcal{L}_{3}=\tilde{\mathcal{B}}_{1}(k)^{T} P \tilde{\mathcal{B}}_{1}(k)+\mathcal{D}_{11}(k)^{T} \mathcal{D}_{11}(k)-\gamma^{2} \mathcal{I}_{n_{\mathbf{d}}} \\
& {\left[\begin{array}{c}
e_{x}(k-\kappa) \\
\mathbf{d}_{[k-\kappa, k)}
\end{array}\right]^{T}\left[\begin{array}{cc}
\mathcal{L}_{1} & \mathcal{L}_{2} \\
\mathcal{L}_{2}^{T} & \mathcal{L}_{3}
\end{array}\right]\left[\begin{array}{c}
e_{x}(k-\kappa) \\
\mathbf{d}_{[k-\kappa, k)}
\end{array}\right] \leq 0 .}
\end{aligned}
$$

Equivalently, we can write

$$
\begin{aligned}
{\left[\begin{array}{cc}
\tilde{\mathcal{A}}(k) & \tilde{\mathcal{B}}(k) \\
\mathcal{C}_{1}(k) & \mathcal{D}_{11}(k)
\end{array}\right]^{T}\left[\begin{array}{cc}
P & 0 \\
0 & \mathcal{I}_{n_{f}}
\end{array}\right] } & {\left[\begin{array}{cc}
\tilde{\mathcal{A}}(k) & \tilde{\mathcal{B}}(k) \\
\mathcal{C}_{1}(k) & \mathcal{D}_{11}(k)
\end{array}\right] } \\
& +\left[\begin{array}{cc}
-P & 0 \\
0 & -\gamma^{2} \mathcal{I}_{n_{\mathbf{d}}}
\end{array}\right] \preceq 0 .
\end{aligned}
$$

Applying the Schur decomposition and a weighted over-bound on $P^{-1}$ by $P-G^{T}-G \succ-G^{T} P^{-1} G$ with $G \succ 0$ [22] we arrive to the matrix inequality in (37).

Remark 1: The disturbance rejection problem can also be formulated as a minimization problem considering $\gamma$ as a decision parameter. Moreover, the inverse system in (18) cannot preserve affinity in the scheduling parameters anymore. Consequently, solution of the feasibility problem in (37) has to be approximated. Instead of solving the disturbance rejection problem $\forall p_{k} \in \mathcal{P}$, we propose to use a gridding based method [18]. Finally, this approximate solution can be performed off-line.

\section{NUMERICAL EXAMPLE}

The above inversion based and disturbance attenuating fault estimator method is illustrated on a simulation example. A third-order discrete-time LPV model of a unbalanced, nonlinear dc motor [23] is used in the sequel.

Given the discrete-time state-space LPV representation by

$$
\begin{aligned}
& {\left[\begin{array}{c}
\theta(k+1) \\
\omega(k+1) \\
I(k+1)
\end{array}\right]} \\
& =\left[\begin{array}{ccc}
1 & T_{s} & 0 \\
p_{k} \cdot T_{s} M g \ell / J & 1-T_{s} b / J & T_{s} K / J \\
0 & -T_{s} K / L & 1-T_{s} R / L
\end{array}\right]\left[\begin{array}{c}
\theta(k) \\
\omega(k) \\
I(k)
\end{array}\right] \\
& +\left[\begin{array}{c}
0 \\
0 \\
T_{s} / L
\end{array}\right] u(k)+\left[\begin{array}{c}
0.1 \\
0.1 \\
0
\end{array}\right] d(k)+\left[\begin{array}{c}
0 \\
p_{k} \cdot \tau \\
0
\end{array}\right] f(k)
\end{aligned}
$$
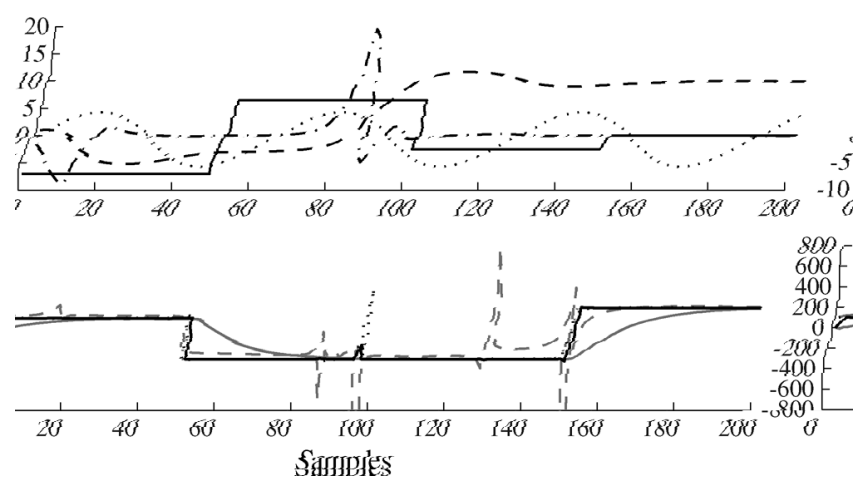

Fig. 1. Simulation results. The first row depicts the $u(k)$ (dotted line), $y(k)$ (dashed and dashdot lines) and $1000 \cdot d(k)$ (solid line). The second row is a comparative plot of nominal inversion based (grey dashed line), robust inversion based with parameter independent (dotted line) and dependent (solid line) gains, and existing robust fault estimation (grey solid line) methods.

$$
\begin{aligned}
& y(t) \\
& =\left[\begin{array}{ccc}
1 & 0 & 0 \\
0 & p_{k} & 0
\end{array}\right]\left[\begin{array}{c}
\theta(k) \\
\omega(k) \\
I(k)
\end{array}\right]+\left[\begin{array}{c}
0.05 \\
0.05\left(1+p_{k}\right)
\end{array}\right] d(k)
\end{aligned}
$$

where $\tau=T_{s} M g \ell$. In this specific case study the following dimensions are used $n_{p}=2, m=1, n_{f}=1, n=3, \ell=2, n_{d}=1$.

By using the nominal motor parameters [23], it can easily be seen that a matrix representation of $\mathcal{C}$ can be written as $\left[\begin{array}{lll}0 & 0 & 1\end{array}\right]^{T}$. We propose to use $E=\left[\begin{array}{lll}0 & -1 & 0\end{array}\right]^{T}$. Based on [14] and $V^{*}=\left[\begin{array}{lll}0 & 0 & 1\end{array}\right]^{T}$, the largest controllable subspace in $\mathcal{C}$ results in $\mathcal{R}^{*}=\{0\}$. The coordinate transformation matrix can therefore be written as $T=\left[V^{*, \perp} V^{*}\right]=$ $\left[\begin{array}{lll}0 & 1 & 0 \\ 1 & 0 & 0 \\ 0 & 0 & 1\end{array}\right]$. Applying $T$ on the system above and computing the feedback gains results in $F_{2}^{(1)}=13.4$ and $F_{2}^{(2)}=0$. Afterwards, the relative degree vector computation returns with $\tilde{\kappa}=\left[\begin{array}{ll}1 & 1\end{array}\right]^{T} \forall p_{k} \in \mathcal{P}$, consequently $\tilde{n}_{d}=2$.

Being $p_{k} \in[0.11]$, an equidistant grid is proposed over $\mathcal{P}$ by 10 partitions $(|\Delta p| \leq 0.05)$. Evaluating the matrix inequality conditions around those grid-points and solving the performance minimization problem, two different disturbance rejection levels were obtained, $\gamma_{1}=$ 1169.1 and $\gamma_{2}=1077.4$. These levels refer to the robust inversion based solutions computed for the cases when the gain matrix is parameter independent $\mathcal{K}$ and parameter varying $\mathcal{K}(k)=\mathcal{K}_{1}+p_{k} \mathcal{K}_{0}^{2}+$ $p_{k-1} \mathcal{K}_{1}^{2}$, respectively.

Fig. 1 depicts the simulation results of a 200 sample long time scenario. The aim of the simulation is to show the viability of the robust method when the fault variation to be estimated is described as

$$
f(k)=\left\{\begin{array}{cc}
100 & \text { if } k \leq 50 \\
-300 & \text { if } 50 \leq k \leq 150 \\
200 & \text { if } 150 \leq k
\end{array}\right.
$$

Methods have been tested and compared when a series of step disturbance act upon the dc motor system. Results of a robust model-based technique derived form [21] is compared to a nominal and robust dynamic inversion estimation techniques. The first row of the plots in Fig. 1 shows the values of the signals $u(k), y(k)$ and $d(k)$. Simulation results in the second row of Fig. 1 depict the achieved results with the nominal and robust inversion based methods, and with the existing robust model-based fault estimator, respectively. Comparing the nominal and robust inversion based results, one can conclude that the ro- 
bust solution attenuates the effect of disturbance more than the nominal one. Robust inversion based techniques with parameter varying gain, $\mathcal{K}(k)$ slightly over-performs the observer with constant gain matrix $\mathcal{K}$ in terms of disturbance rejection, which can also be seen at time sample 96 (differences between dotted and solid lines). Contrasting the robust inversion based alternatives (with $\mathcal{K}$ and $\mathcal{K}(k)$ ) for FD to the existing and model-based robust observer design (grey solid line), one can see that the robust inversion based solution could react faster to show the presence of the fault, although, in this specific case the existing technique was more robust against disturbances.

\section{CONCLUSION}

The technical note shows a novel and robust algorithm for discretetime LPV fault estimation. This method is proposed to use inversion based concepts and to describe how to attenuate an additive disturbance signal on the fault reconstruction error in an induced $\mathcal{L}_{2}$ norm sense. Dynamic inversion w.r.t. additive fault input does not preserve the originally affine parameter varying structure, and introduces (relative degree dependent) time delay in the inverted system description. In case of an additive and exogenous disturbance signal, a robust objective can be formulated. With the help of a Luenberger fault observer, the technical note presented a robust and inversion based LPV FD method. Since the availability of fault-free scheduling signal is of paramount importance for the underlying design process, further research can be carried out to investigate the effect of impair scheduling measurement.

\section{APPENDIX}

Algorithm 2 (Relative Degree Vector Computation): Denote by $c_{i}^{(j)}$ the $i^{\text {th }}$ row of $\tilde{C}_{2}^{(j)}, i=1 \ldots \ell, j=1 \ldots n_{p}$. Given the system in (9)-(10), the relative degree $\kappa_{i}$ associated to the output channel $i$ w.r.t the fault input $E_{1}$ can be algorithmized as, $S_{i}^{0}=\sum_{j=1}^{n_{p}} p_{k}^{(j)} c_{i}^{(j)}$, $S_{i}^{1}=\sum_{j=1}^{n_{p}} p_{k+1}^{(j)} c_{i}^{(j)} \sum_{j=1}^{n_{p}} p_{k}^{(j)} A_{11}^{(j)}, S_{i}^{\kappa_{i}}=\overrightarrow{S_{i}{ }^{\kappa_{i}-1}} \sum_{j=1}^{n_{p}} p_{k}^{(j)} A_{11}^{(j)}$, where $\overrightarrow{(\cdot)}$ denotes the forward shift operator, until we reach $\kappa_{i}$ where $\overrightarrow{S_{i}{ }^{\kappa_{i}}} \sum_{j=1}^{n_{p}} p_{k}^{(j)} E_{1} \neq 0$ but $\forall l<\kappa_{i}, \overrightarrow{S_{i}{ }^{l}} \sum_{j=1}^{n_{p}} p_{k}^{(j)} E_{1}=0 \forall p_{k} \in \mathcal{P}$. Performing this computation to all output channel, we can define the relative degree vector $\tilde{\kappa}=\left[\kappa_{1} \ldots \kappa_{\ell}\right]$. The maximal element of the vector will be denoted by $\kappa=\max (\tilde{\kappa})$.

\section{ACKNOWLEDGMENT}

The authors thank the anonymous reviewers for helpful comments, and Aeje.

\section{REFERENCES}

[1] J. Gertler, Fault Detection and Diagnosis in Engineering Systems. New York: Marcel Dekker, 1998.

[2] M. Blanke, M. Kinnaert, J. Lunze, and M. Staroswiecki, Diagnosis and Fault-Tolerant Control. Berlin, Germany: Springer Verlag, 2003.

[3] J. Chen and R. Patton, Robust Model-Based Fault Diagnosis for Dynamic Systems. Boston, MA: Kluwer Academic Publisher, 1999.

[4] S. X. Ding, Model-Based Fault Diagnosis Techniques. Berlin, Germany: Springer Verlag, 2008.

[5] J. Bokor and Z. Szabó, "Fault detection and isolation in nonlinear systems," Annu. Rev. Control, vol. 33, no. 2, pp. 113-123, 2009.

[6] M. Staroswiecki and G. Comtet-Varga, "Analytical redundacy relations for fault detection and isolation in algebraic dynamic systems," Automatica, vol. 37, no. 5, pp. 687-699, 2001.

[7] C. De Persis and A. Isidori, "A geometric approach to nonlinear fault detection and isolation," IEEE Trans. Autom. Control, vol. 46, no. 6, pp. 853-856, Jun. 2001.

[8] J. Shamma and M. Athans, "Guaranteed properties of linear parameter varying gain scheduled control systems," Automatica, vol. 27, no. 3, pp. 559-564, 1991.
[9] J. Bokor and G. Balas, "Detection filter design for LPV systems-A geometric approach," Automatica, vol. 40, no. 3, pp. 511-518, 2003.

[10] D. Henry, A. Falcoz, and A. Zolghadri, "Structured $\mathcal{H}_{\infty} / \mathcal{H} \_$LPV filters for fault diagnosis: Some new results," in Proc. IFAC Safeprocess, Barcelona, Spain, 2009, pp. 420-425.

[11] S. Armeni, A. Casavola, and E. Mosca, "Robust fault detection and isolation for LPV systems under a sensitivity constraint," Int. J. Adaptive Control Signal Processing, vol. 23, no. 1, pp. 55-72, 2009.

[12] G. F. A. Casavola, D. Famularo, and M. Sorbara, "A fault-detection, filter-design method for linear parameter-varying systems," Proc. IMechE Part I., J. Syst. Control Eng., vol. 221, no. 6, pp. 865-873, 2007.

[13] Z. Szabó, J. Bokor, and G. Balas, "Inversion of LPV systems and its application to fault detection," in Proc. IFAC Safeprocess, Washington, DC, 2003, vol. 1, pp. 235-240.

[14] G. Balas, J. Bokor, and Z. Szabó, "Invariant subspaces for LPV systems and their applications," IEEE Trans. Autom. Control, vol. 48, no. 11, pp. 2065-2069, Nov. 2003.

[15] B. Kulcsár, J. Bokor, and J. Shinar, "Unknown input reconstruction for LPV systems," Int. J. Robust Nonlin. Control, vol. 20, no. 5, pp. 579-595, 2010.

[16] M. Fliess, "Reversible linear and nonlinear discrete-time dynamics," IEEE Trans. Autom. Control, vol. 37, no. 8, pp. 1144-1153, Aug. 1992.

[17] C. Gori-Giorgi and S. Monaco, "Uniformity and reversibility conditions for linear time varying systems," Ricerche di Automatica, vol. 8, no. 2-3, pp. 142-151, 1977.

[18] F. Wu, X. Yang, A. Packard, and G. Becker, "Induced $\mathcal{L}_{2}$ norm bounded control for LPV systems with bounded parameter variation rates," Int. J. Robust Nonlin. Control, vol. 6, no. 9-10, pp. 983-998, 1996.

[19] W. Wohnam, Linear Multivariable Control-a Geometric Approach. New York: Springer Verlag, 1985.

[20] P. Bolzern, P. Colaneri, and G. De Nicolao, "On discrete-time $\mathcal{H}_{\infty}$ fixed-lag smoothing," IEEE Trans. Signal Processing, vol. 52, no. 1, pp. 132-141, Jan. 2004.

[21] Z. Weng, R. Patton, and P. Cui, "Robust fault estimation for linear parameter varying time delay systems," in Proc. 16th Med. Conf. Control Autom., Ajaccio, France, 2008, pp. 292-296.

[22] M. de Oliveira, J. Bernussou, and J. Geromel, "A new discrete-time robust stability condition," Syst. Control Lett., vol. 37, no. 4, pp. 261-265, 1999.

[23] B. Kulcsár, J. Dong, J. van Wingerden, and M. Verhaegen, "LPV subspace identification of a dc motor with unbalanced disc," in Proc. 15th IFAC Symp. Syst. Ident., Saint-Malo, France, 2009, pp. 856-871. 